\title{
INNOVACIÓN EN LA TRANSFORMACIÓN PRODUCTIVA INDUSTRIAL: APORTES A LA DISCUSIÓN*
}

La inspiración existe pero tiene que encontrarte trabajando.

PICASSO

\author{
Marleny Cardona A.** \\ Santiago Escobar . $^{* * *}$
}

Recibido: marzo 30 de 2011 • Aceptado: mayo 23 de 2012

\section{RESUMEN}

El propósito de este artículo es identificar elementos y características de la innovación empresarial que se tienen en cuenta en la política de transformación productiva en el país. La estrategia metodológica empleada para lograr este propósito fue la revisión, sistematización y análisis de artículos de acuerdo con las categorías innovación y transformación productiva. Como principal resultado este análisis se encontró que la innovación es un eje fundamental de la transformación productiva, porque orienta el fortalecimiento industrial con apoyo al desarrollo de las empresas; sin embargo, su inserción ha sido débil. A través de ella se visibilizan los cambios y se constituyen dinámicas que enlazan actividades e integran la diversidad de culturas, a través de las cuales se acumula conocimiento y se garantizan competencias individuales y colectivas que se transforman en potencialidades sociales para las empresas.

\section{PALABRAS CLAVE}

Innovación, políticas industriales y regulación, innovación social.

\section{CLASIFICACIÓN JEL} O31, O33, L52.

\section{CONTENIDO}

Introducción; 1. Innovación: carácter social y capacidades humanas; 2. Transformación productiva: desarrollo productivo y mercados internacionales; 3. Conclusiones; Bibliografía.

- Este artículo de investigación es producto del proyecto "Sistemas de conocimiento e innovación en las PyMEX exportadoras de los sectores alimentos (biotecnología) y textil/confección/diseño/moda en BogotáSoacha, en el período 1990-2007", adscrito al Centro de Investigaciones de la Escuela de Negocios, Fundación Universitaria Konrad Lorenz (FUKL), Bogotá, Colombia. Grupo de investigación Transformación productiva, desarrollo local y competitividad, clasificación C de Colciencias. El proyecto fue desarrollado en el período 2010-2011.

** Economista de la Universidad de Antioquia, Medellín, Colombia. Especialista en Planeación Urbana y Regional, Universidad Nacional, Medellín, Colombia. Doctora en Ciencias Sociales, Colegio de la Frontera Norte, Tijuana, México. Directora del Centro de investigaciones de la FULK, Bogotá, Colombia. Miembro del Grupo de Investigación Desarrollo Local, Transformación Productiva y Competitividad, Bogotá, Colombia. Correo electrónico: marleny.cardona@konradlorenz.edu.co.

*. Economista Universidad Jorge Tadeo Lozano, Bogotá, Colombia. Especialista en docencia Universidad El Bosque, Bogotá, Colombia. Curso Internacional en Desarrollo económico local en Cerur, Rehovoth, Israel. Profesor de cátedra del centro de investigaciones FULK, Bogotá, Colombia. Miembro del Grupo de Investigación Desarrollo Local, Transformación Productiva y Competitividad, Bogotá, Colombia. Correo electrónico: pastors.escobara@konradlorenz.edu.co. 


\title{
INNOVATION IN THE PRODUCTIVE INDUSTRIAL TRANSFORMATION: CONTRIBUTIONS TO THE DISCUSSION
}

\section{ABSTRACT}

The goal of this paper is to identify elements and characteristics of business innovation that are part of the country's (Colombia) Productive Transformation Policy. The method used to achieve this goal was to review, systematize and analyze articles according to the innovation and productive transformation categories. As its main result, it was found that innovation is a fundamental tool for productive transformation because it focuses on industrial strengthening, supporting the development of business; however, its implantation has been limited. Through it, changes are visualized and dynamics put in place that link activities and integrate cultural diversity by which knowledge is accumulated and individual and group competences are developed that become social powers for businesses.

\section{KEY WORDS}

Innovation, industrial and regulation policies, social innovation

\section{JEL CLASSIFICATION}

O31, O33, L52.

\section{CONTENT}

Introduction; 1. Innovation: social character and human capabilities; 2. Productive transformation, productive development and international markets; 3. Conclusions; Bibliography

\section{INOVAÇÃO NA TRANSFORMAÇÃO PRODUTIVA INDUSTRIAL: APORTES À DISCUSSÃO}

\section{RESUMO}

O propósito deste artigo é identificar elementos e caraterísticas da inovação empresarial que são levadas em conta na política de transformação produtiva no país. A estratégia metodológica empregada para conseguir este propósito foi a revisão, sistematização e análise de artigos segundo as categorias de inovação e transformação produtiva. Como principal resultado desta análise se encontrou que a inovação é um eixo fundamental da transformação produtiva, porque orienta o fortalecimento industrial com apoio ao desenvolvimento das empresas; porém, sua inserção tem sido débil. Por meio dela se visualizam as mudanças e se constituem dinâmicas que enlanzam atividades e integram a diversidade de culturas, a través das quais se acumula conhecimento e se garantem competências individuais e coletivas que se transformam em potencialidades sociais para as empresas.

\section{PALAVRAS-CHAVE}

Innovación, políticas industriales y regulación, innovación social.

\section{CLASSIFICAÇÃO JEL}

\author{
O31, O33, L52.
}

\section{CONTEÚDO}

Introdução; 1. Inovação: caráter social e capacidades humanas; 2. Transformação produtiva: desenvolvimento produtivo e mercados internacionais; 3. Conclusões; Bibliografia. 


\section{INTRODUCCIÓN}

El carácter social de la innovación se refleja en la capacidad de las empresas para la incorporación del conocimiento en la transformación productiva ${ }^{1}$. Es desde la innovación que las decisiones de los agentes privados o públicos con el apoyo desde las políticas públicas llevan al desarrollo productivo. La incorporación en la sociedad del conocimiento requiere dos aspectos: la productividad y la competitividad, por un lado, dependen de la capacidad de adquirir conocimiento y de procesar información, lo cual quiere decir educación, adaptada a ese nuevo tipo de producción basado en la generación de conocimiento; por el otro, requiere de articulaciones en red, esto quiere decir que cada vez más las empresas y todo tipo de organizaciones funcionan en su práctica cotidiana en redes.

Este trabajo tiene como propósito identificar elementos y características de la innovación empresarial que se tienen en cuenta en la búsqueda de la transformación y estructuración productiva en el país; para ello, se elaboró una revisión documental de las políticas y estudios referentes al tema; estos fueron sistematizados a través de fichas textuales y analíticas para identificar los hallazgos de cada investigación y los postulados de los autores frente a la innovación y transformación productiva.

El desarrollo productivo es una construcción social, resultado de las dinámicas entre proveedores, productores y consumidores en un territorio; no es asunto aislado de las empresas. De esta reflexión surgen en este trabajo las siguientes preguntas: ¿Están relacionadas las capacidades de innovación y tecnologías con el desempeño de las empresas colombianas? ¿Cuáles de dichas capacidades y con qué tipo de desempeño? En particular, interesa de acuerdo con Robledo, Gómez y Restrepo (2008, p. 2), explorar la relación entre las capacidades de innovación y tecnologías y el desempeño empresarial medido desde dos ópticas: una enfocada al desempeño innovador, el cual está vinculado de forma directa con las capacidades de innovación tecnologías, y otra enfocada al desempeño del negocio, relacionado este con el impacto de la innovación en las ventas, las utilidades, la participación en el mercado y las exportaciones.

Los temas se clasificaron a través de tres categorías: 1) la innovación desde el carácter social y las capacidades humanas; 2) la transformación productiva en el contexto del desarrollo productivo y mercados internacionales; 3) Tecnologías y desarrollo de productos, las cuales incorporan la compresión desde la economía global.

Como principales hallazgos se encuentran la interacción entre el espacio global de las firmas y los sistemas locales, que de cierto modo, esperan ser potenciados

La transformación productiva se ha vuelto un tema de política en las economías. En Colombia es un Programa que se inicia desde 2006, que busca el acercamiento empresa-Estado para el desarrollo sectorial desde el desarrollo empresarial. La base del programa es la política de competitividad. 
con la política de transformación productiva en la medida en que se promueven las capacidades desde las oportunidades de las firmas al articular los dos entornos en el ámbito del conocimiento.

Este documento está estructurado para dar al lector una dimensión general de los conceptos y políticas clave de la transformación productiva y la innovación para su posterior evaluación. El artículo está divido en tres secciones: la primera, Innovación: Carácter social y capacidades humanas; la segunda, Transformación productiva: Programas, políticas y mercados internacionales y, por último, las conclusiones.

\section{INNOVACIÓN: CARÁCTER SOCIAL Y CAPACIDADES HUMANAS}

La innovación es jalonada desde las grandes empresas, porque estas descentralizan procesos que desarrollan en muchos casos las pymes y en otros los distintos departamentos y divisiones de la empresa ${ }^{2}$. Las pequeñas y medianas funcionan en conjunto, organizadas en redes, esto les permite desarrollar los recursos que por sí solas no podrían poseer, y a la vez, trabajar para grandes empresas descentralizadas en redes internas. Desde la perspectiva de los recursos y capacidades, se entiende que detrás de la innovación, es inherente el proceso empresarial, que expresa las capacidades organizacionales y funcionales que la hacen viable y posible.

La transformación productiva, según Robledo, Gómez y Restrepo (2008) y Christesen (1995), sustentada en procesos de innovación se logra con capacidades, en especial las que tienen que ver con la producción, espacio en el cual se expresan los resultados de las capacidades humanas y técnicas. Es así como la socialización intra e interempresarial de los procesos productivos es el eje fundamental; esta da cuenta del fenómeno de la difuminación o spillovers como mecanismos fundamentales para la incorporación de la innovación. Schumpeter (1978) explica que el sistema económico puede caer en una especie de destrucción creativa donde las nuevas tecnologías, producto de la innovación, acelera los cambios que se evidencian en las empresas.

El carácter social de la innovación hace que la transformación productiva tenga un efecto público y privado, que se traduce en incrementos de productividad y competitividad para las empresas y la sociedad. La relación entre innovación y transformación productiva da cuenta de las condiciones socioeconómicas y culturales en las cuales se produce e incorpora el conocimiento. El desarrollo de nuevos productos y servicios se realiza en forma endógena en una firma o en un territorio; para ello se requieren competencias especializadas, que respondan a una lógica de extensión progresiva e incremento de la experiencia. Además, cuando se buscan salidas comerciales hacia otros dominios se produce la capacidad de innovación

Las empresas se clasifican según el número de empleados, conforme a lo establecido en la Ley 590 de 2000 (CR, 2000), modificada por la Ley 905 del 2004 (CR, 2004). 
basada en la experiencia acumulada: experiencia cruzada entre productores, entre productores y clientes y entre clientes, que garantizan el incremento de la productividad y la competitividad.

En el Esquema 1, el conocimiento como proceso que tiene como resultado la innovación depende en especial del progreso científico, en la combinación de aportes de las disciplinas con conocimientos técnicos, y desarrollos de la innovación que evidencian resultados de la inversión en Investigación y Desarrollo (I+D). Se necesitan relaciones empresariales en red, fuerza de trabajo calificada, infraestructuras y servicios que funcionen de forma eficiente, empresas que tengan capacidad para adoptar, adaptar y aplicar conocimientos que se han generado fuera de ellas; de ahí el carácter social, que facilita procesos de información y aprendizaje que operan como empresas articuladas a la producción desde subcontratistas, proveedoras, clientes y usuarios.

Estas articulaciones a redes industriales generan transferencia tecnológica y competencias laborales que garantizan el espacio de la innovación en los sistemas productivos. Las redes dan cuenta de las articulaciones, confianza e intercambio entre las empresas. En el Esquema 1, la segunda dimensión sobre transferencia tecnológica expresa los resultados de los procesos innovadores entendidos como la aplicación de los nuevos conocimientos y la socialización de los mismos. Y, por último, en las competencias laborales se garantiza que la transferencia tecnológica se asimile y se puedan articular los resultados en un producto, proceso o servicio (Cardona y Osorio, 2003). A manera de ejemplo, el límite de los esquemas innovadores está en las dinámicas productivas y el tejido social para el desarrollo del producto o servicio; según Veltz (1999), el problema es el riesgo de encerrarse en un conjunto limitado de productos y procedimientos.

Esquema 1. Elementos para la innovación con dinámica empresarial e integración del conocimiento

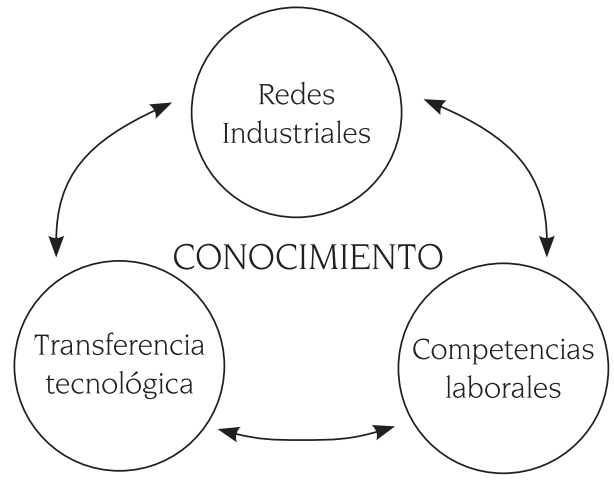

Fuente: Tomado de Cardona y Osorio (2003, p. 25) 
Otro punto asociado a la innovación como resultado de la investigación y el desarrollo desde organizaciones públicas y privadas es la expresión de la dinámica social que se teje en los sistemas productivos a través de los cuales se logra que las transformaciones en el proceso den cuenta de la dinámica de los factores, capital, trabajo y tierra. En este sentido Robledo, Gómez y Restrepo (2008) retoman autores como Yam y otros (2004) para exponer sobre las capacidades que se requieren para generar procesos de desarrollo con innovación que dependen de las capacidades expresadas en habilidades y desempeño en uso de recursos:

- Capacidad de gestión de recursos: se refiere a la habilidad de las organizaciones para adquirir y asignar de forma apropiada capital, experiencia y tecnología a los procesos de innovación.

- Capacidad de aprendizaje organizacional: esta capacidad posibilita la adquisición de conocimiento implícito y explícito, el traspaso de conocimiento y el uso del conocimiento. Además, se reconoce como la habilidad del establecimiento para identificar, asimilar y explotar el conocimiento proveniente del ambiente circundante. Desde otra óptica, DiBella, Nevis y Gould (1996), la definen como la habilidad de mantener o mejorar el desempeño basado en la experiencia; con ello se refieren en forma particular al learning-by-doing.

- Capacidad de planeación estratégica: para Yam y otros (2004) esto hace parte de la destreza de una empresa para reconocer los factores externos, debilidades y fortalezas; los internos, amenazas y oportunidades y lograr articularlos a su plan de forma coherente con los objetivos corporativos; mientras que Guan y Ma (2003) la definen como la capacidad para sobresalir en los ambientes competitivos actuales.

- Capacidad de producción: la habilidad de la empresa para transformar los resultados de investigación y desarrollo en productos que satisfagan los requerimientos del mercado, al integrar los requisitos del diseño y las limitaciones y posibilidades del sistema de manufactura disponible para la empresa.

- Capacidad de mercadeo: esta capacidad representa la habilidad de la firma para publicitar y vender productos de acuerdo con la comprensión de las necesidades del mercado tanto presentes como futuras, el ambiente competitivo, los costos y beneficios, y la aceptación de la innovación.

- Capacidad organizacional: la habilidad de la empresa para asegurar el mecanismo y la armonía organizacional, al promover la cultura organizacional y en la adopción de buenas prácticas de gestión.

Entre estas capacidades que son vistas como habilidades se incorporan a los procesos productivos en forma individual y colectiva con mayor facilidad los que tienen que ver con producción, mercadeo y planeación estratégica. A su vez, es el 
conocimiento el que se plasma en la innovación como un proceso de aprendizaje que requiere mayor especialización. Cohen y Kepler (1996) plantean la innovación soportada en el clúster como sistema territorial; allí se crea una mutua asociación, se da apropiación de la maquinaria para mantener empresas innovadoras, representadas en comunidades de interés económico con aprovechamiento de las ventajas económicas de establecer un mercado unificado y liderado por los mismos. Estas capacidades se desarrollan mediante la incorporación de procesos y técnicas de gestión para recopilar información pública; de igual forma se introduce el concepto de capacidades de innovación tecnológica (CIT); se refiere a capacidades genéricas y específicas en las empresas, aunque necesita que se lleven a cabo de manera simultánea criterios de orden cuantitativo y cualitativo aplicados a la organización (Robledo, Gómez y Restrepo, 2008, p. 2).

Para concluir, la innovación como concreción del conocimiento permite que la acumulación de conocimiento desde las capacidades no sea un fin en sí mismo, sino más bien un medio para lograr ciertos objetivos en los sistemas productivos y empresariales. El logro de tales objetivos corresponde a lo que se denomina aquí desempeño empresarial, definido desde dos ópticas: 1) el desempeño Innovador, entendido como el logro de innovaciones tecnológicas de producto y proceso; y 2) el desempeño del Negocio, relacionado con el impacto de la innovación en las ventas, las utilidades, la participación en el mercado y las exportaciones.

Asimismo, la innovación es el resultado de la incorporación de las capacidades humanas junto a las capacidades productivas y organizacionales. La transformación productiva se garantiza en los procesos, acciones y políticas establecidas para su desarrollo con incorporación de conocimiento. En una revisión crítica de la literatura, se muestra que existe una especial atención sobre la cuestión de si las organizaciones pueden adaptarse a los cambios tecnológicos y ambientales, o si la transformación de la forma de organización se produce en particular por las competencias laborales y la innovación. De ahí, que la preocupación sea por los sistemas locales para el desarrollo productivo articulados a través de los agentes locales en búsqueda de la competitividad.

La incorporación de elementos estructurales en el análisis de los procesos productivos y organizacionales muestra la relación en la dinámica de la organización con la innovación desde tres perspectivas interdependientes: a) la relación entre las formas de organización estructural y la innovación, b) la innovación como un proceso de aprendizaje organizacional y creación de conocimiento, y c) la capacidad de la organización para el cambio y la adaptación.

El desarrollo de la cultura innovadora tiene como antecedente potenciar las capacidades de creación de los individuos y las organizaciones productivas, donde 
las políticas educativas para el trabajo, sustentadas en el derecho al desarrollo, garantizan a los países y en particular a las organizaciones, tener ventaja competitiva sostenida, desde el uso de los recursos que obtienen de su entorno. Así, las capacidades humanas hacen que se supere el supuesto de que el hecho de que un país tenga una ventaja competitiva en una industria no implica que las empresas en esa industria sean en el campo internacional competitivas, ni implica que el país sea competitivo en el ámbito internacional.

En Robledo, Gómez y Restrepo (2008), y Yam y otros (2004), se identifican las capacidades organizacionales asociadas con la innovación tecnológica; en los encadenamientos de la capacidad investigación más desarrollo (I+D), es la habilidad de la firma para integrar los factores y construir esta estrategia, la implementación de proyectos, la gestión de portafolios de proyectos y los gastos de I+D. La tecnología, el capital y las instituciones son dimensiones para garantizar mercados que tiene su centro en la adaptación de los productos. Los mercados de factores estratégicos externos, por un lado, proveen fuentes nacionales específicas de ventaja y, por el otro, los factores estratégicos internos proveen fuentes específicas para la empresa. Los primeros son valiosos para las empresas a través del poder de monopolio, mientras que los segundos, solo se vuelven valiosos cuando se internalizan a través de las actividades de valor de la empresa, lo que crea heterogeneidad entre las empresas $y$, por lo tanto, da ventajas competitivas.

La innovación para el desarrollo de capacidades organizativas puede ser una condición previa necesaria para la innovación tecnológica, y por lo tanto es importante tener en cuenta el papel de las fuerzas endógenas de la organización, tales como la capacidad para el aprendizaje, los valores, los intereses y el poder, los cuales se requiere en la configuración de la transformación organizacional con el cambio tecnológico, como lo plantean Lam (2002), Castell y Hall (2001), Castell (2002), Duran y otros (1998) y Audretcsh y Feldman (1996). Además, las teorías de la organización, las teorías de la adaptación estratégica y el cambio continuo son referentes para la comprensión del problema.

La innovación es una dimensión estructural, por ser un espacio de desarrollo productivo (ver esquema 2), se orienta al fortalecimiento empresarial en especial de las pymes con apoyo de la política Nacional desde el Consejo Asesor de Ciencia Tecnología e Innovación (CTI) y de lineamientos institucionales. Este Consejo es el encargado de asesorar a Colciencias, sugerir los criterios para la calificación de programas y proyectos en materia de CTI, asesorar sobre los programas, políticas, planes y proyectos estratégicos para el desarrollo científico y tecnológico del país, entre otras. 
Esquema 2. Dimensiones de la innovación para la transformación productiva

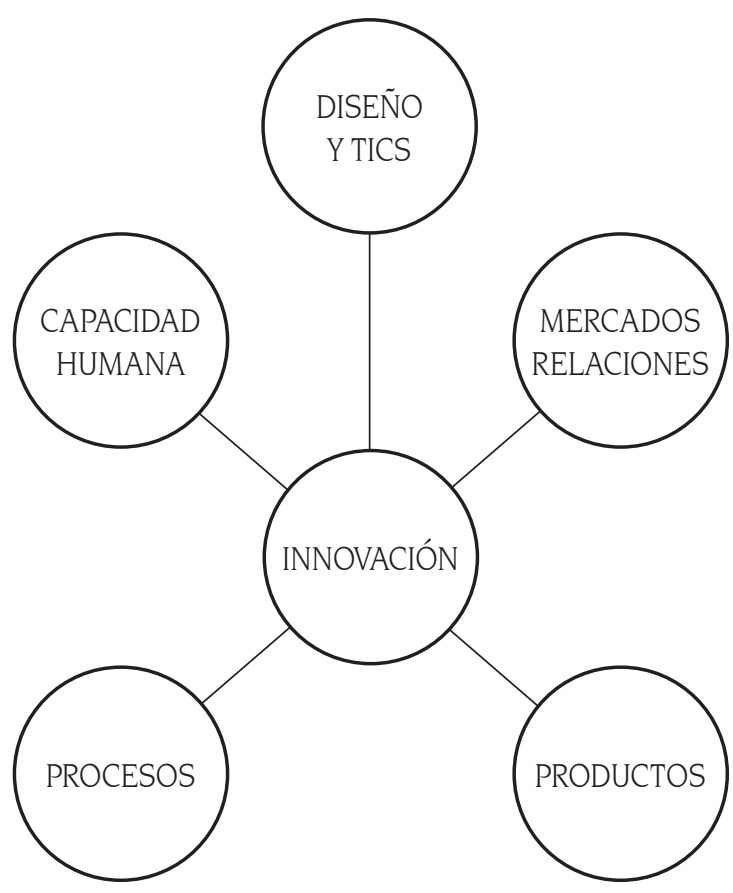

Fuente: elaboración propia

Las dimensiones de la innovación tienen un carácter social que se expresa en las formas productivas locales. La propuesta de Ciencia y Tecnología tiene su eje principal en el apoyo a la empresa, en esta se han definido incentivos que representan, según DNP (2007), entre 2002 y 2010 beneficios por más de un billón de pesos para impulsar proyectos empresariales de desarrollo tecnológico e innovación. Además, para el año 2011, se encuentra en trámite una reforma que agilizará el acceso del sector productivo a la política de transformación productiva, que considera la in novación como el proceso de proposición, adopción, desarrollo e implementación de una nueva idea, generada en el ámbito interno o tomada del exterior relacionada con un producto, proceso, política, práctica o comportamiento, programa o servicio que es nueva para la organización en el momento de la adopción y que beneficiará a la misma o a la sociedad en general.

El gobierno colombiano estimula a través del uso de instrumentos tributarios como herramienta para el desarrollo de sectores específicos y el crecimiento productivo territorial a partir del gobierno 2002-2010, DNP (2007a, p. 244). Es más, según Galindo y Meléndez (2011), de acuerdo con el Conpes 3322 de 2004 (DNP, 2004), las 
exenciones al impuesto a la renta ${ }^{3}$ que fueron concedidos a sectores o actividades específicas se calculó en el orden de 1.41\% del PIB (1,520 millones de dólares).

Asimismo, en el Conpes 3484, DNP (2007, p. 5), el desarrollo productivo se fundamentó en garantizar los recursos crediticios para aumentar, por un lado, las capacidades productivas y organizativas para la innovación como estrategia en la creación de un modelo sectorial de clase mundial; y por el otro, se promovieron desde el Plan Nacional de Desarrollo 2006-2010, DNP (2007a, p.244), incentivos a la Inversión Extranjera Directa. Además, está el fomento interno el Consejo Privado de Competitividad, CPC (2011, p. 179 181), el cual promueve varios tipos de estímulos tributarios como la reducción de la tarifa del impuesto sobre la renta; reducción de la carga tributaria sobre la nómina; eliminación del impuesto al patrimonio para personas jurídicas; eliminación de exenciones, deducciones y descuentos; reducción del número de tarifas de IVA; alcance del régimen simplificado del IVA; ajuste en tarifas de retención en la fuente y ampliación de la base tributaria.

A manera de ejemplo, en el 2007, se aprobaron 83 solicitudes de estímulos tributarios por un valor total de $\$ 134.800$ millones de pesos, mientras que en todo 2009 se aprobaron 73 solicitudes por $\$ 64$ mil millones de pesos DNP (2007, p. 5). El Consejo está conformado por miembros de la academia, el sector productivo y el gobierno, y ha contribuido a una mayor eficiencia en la aprobación de los beneficios que incentivan la investigación y la innovación empresarial.

La cultura empresarial para la innovación se orienta a aumentar la sostenibilidad de las empresas y la competitividad. Desde esta visión las categorías o áreas de las empresas innovadoras siguen un proceso que tiene que ver con la orientación

3 De acuerdo con la DIAN (2008, p. 12) existen cuatro tipo de exenciones importantes de incluir en este análisis:

a. La deducción por inversión en activos fijos reales productivos adquiere el carácter de incentivo permanente. El aumento en el porcentaje objeto de la deducción por inversión en activos fijos, que pasa del 30\% al 40\% para el año 2007...;

b. La creación del descuento por inversión en acciones de sociedades agropecuarias; consiste en que los contribuyentes que inviertan en acciones de empresas agropecuarias, tienen derecho a descontar el valor de la inversión realizada, sin que exceda del uno por ciento (1\%) de la renta líquida gravable obtenida el año en el cual se realiza la inversión (Art. 249 E.T.)...

c. Modificación en la aplicación del descuento por impuestos pagados en el exterior. La reforma permite que los contribuyentes nacionales que perciban dividendos o participaciones recibidas de sociedades domiciliadas en el extranjero, sujetos al impuesto sobre la renta en el país de origen, que no tengan un acuerdo o convenio de integración, puedan descontar el monto del impuesto en cabeza de la sociedad emisora. Es decir, para que proceda este descuento no se requiere que la sociedad de la cual se recibe el dividendo esté domiciliada en un país con el cual Colombia tenga suscrito un acuerdo o convenio de integración (Art. 254 del E.T)...

d. Eliminación del impuesto de remesas. Antes de la última reforma tributaria, las empresas extranjeras que remesaban a su casa matriz las utilidades obtenidas por su sucursal en Colombia, debían pagar a título de impuesto de remesas el 7\% de las utilidades giradas. 
de la innovación hacia las siguientes actuaciones y acciones: dirección de la innovación, a través de guías o cartas de navegación que garanticen rentabilidad en los desarrollos; apropiación de ideas, mediante la investigación e identificación de mercados; valoración de posibilidades; aprovechamiento al máximo los recursos mediante mecanismos ágiles; capacidad de asociarse y red empresarial, vinculación de la empresa con la investigación en el logro de nuevos productos; legitimación de las ideas, lograr que las ideas sean aprobadas y reconocidas en los bancos de proyectos; dar el mejor uso a los recursos a través de la planificación y el seguimiento a todos los procesos; promover la innovación mediante la difusión organizacional de las metas agregadas e individuales.

En la creación de directrices para unificar criterios en el ámbito internacional se diseñó el Manual de Oslo, OCDE (2006) y Dosi (1982), que incluye el planteamiento de las conexiones y redes entre empresas como potenciadoras de la creación de innovación y la capacidad de aprendizaje y absorción de las empresas. Según la definición del Manual de Oslo, la innovación se refiere a la implementación de un producto, proceso, método organizacional o método de mercadeo nuevo o mejorado en forma significativa OCDE (2006, p. 23). En particular, hasta su segunda edición, el Manual de Oslo hacía referencia en especial a la innovación tecnológica de productos y procesos, y solo en la tercera edición fueron introducidas al Manual las innovaciones organizacionales y en el mercadeo como objeto de medición.

La comprensión de la realidad productiva en la cual se inscribe la innovación pasa por la revisión de autores como Schumpeter (1978), Scherer (1980), Christensen (1995) y Robledo, Gómez y Restrepo (2008). Estos autores amplían la dimensión de la competitividad y productividad como eje fundamental de las explicaciones hacia desarrollos tecnológicos con innovación; esta es necesaria pero no suficiente para lograrlo. Además, según Scherer (1980), es equivocada la búsqueda de un tamaño de empresa que sea óptimo para la innovación, de acuerdo con la doctrina económica clásica y neoclásica que sostiene que las empresas pequeñas, impulsadas por la motivación competitiva, son propicias para el progreso tecnológico, mientras Schumpeter (1978) afirma que las grandes empresas son las mejor dotadas para explotar las oportunidades de innovación. Existen cuatro aspectos fundamentales a analizar: 1) la innovación en procesos y productos (Robledo, Gómez y Restrepo, 2008, p. 2), la evaluación de las capacidades para innovación tecnológica y desempeño (Christensen, 1995, p. 3) el proceso de creación destrucción desde Schumpeter (1978) con nuevos productos, nuevas fuentes de suministro y nuevos tipos de organización y 4) el mercado que pone el contexto propicio para la innovación (Neffa, 2000, p. 25).

En el caso de Colombia se hizo una adaptación del Manual de Oslo, con base en la consulta a empresarios y la medición realizada por Robledo, Gómez y Restrepo 
$(2008)^{4}$ de donde se identifican diferencias conceptuales y de aplicación de variables para la medición:

1. En la primera Encuesta de Desarrollo e Innovación Tecnológica (EDIT), para la medición del desempeño empresarial se utilizó una variable observable desde el valor de las utilidades (o pérdidas) sobre las ventas del establecimiento en 1995; mientras que la segunda encuesta (EDIT) no permite el acceso a datos sobre ventas, utilidades (pérdidas) y exportaciones de la empresa, por lo que no es posible construir variables latentes de desempeño de la unidad productiva.

2. La primera encuesta (EDIT) no presenta datos que puedan relacionarse con la capacidad de mercadeo, por lo que esta variable latente queda sin representación en el modelo.

3. En las dos encuestas faltan datos que puedan relacionarse con la capacidad organizacional; asimismo, los datos existentes se recogen mejor en la variable latente de capacidad de planeación estratégica.

4. La capacidad de aprendizaje organizacional es una variable común en las dos encuestas, asociada a variables observables relativas a la capacitación.

5. En la primera y segunda encuestas (EDIT) se encuentra que falta consistencia, correspondencia y continuidad entre las variables definidas como observables.

6. En los resultados de la aplicación de las dos encuestas se encuentra que los esfuerzos por garantizar espacios para el desarrollo de procesos con innovación tienen respuestas parciales por parte de las empresas acompañadas desde el territorio.

Según Christensen (1995) y Neffa (2000), la capacidad de producir innovaciones tecnológicas había sido asociada a la capacidad de I+D de la empresa. Bajo esta perspectiva, gran parte de la literatura había concentrado su atención en la I+D desde una perspectiva de política y de gestión, como la única actividad capaz de producir innovaciones tecnológicas. Sin embargo, este enfoque tradicional se queda corto frente al amplio espectro de capacidades que se consideran hoy necesarias para la innovación y la tecnología. Además, las capacidades de innovación y tecnologías (CIT) de una empresa comprenden no solo la capacidad de I+D sino también aquellas capacidades ligadas a áreas funcionales de la cadena de valor, a las fortalezas inter-funcionales y generales de gestión de la organización.

La estructura industrial del país se evidencia en la medición que realiza la EDIT, la cual se sustenta en la consulta empresarial, por eso la preocupación por el uso de la misma. Esta tiene que ver con la medición de las capacidades de innovación y

\footnotetext{
Robledo, Gómez y Restrepo (2008, p. 5) hacen mediciones de acuerdo con el análisis de las encuestas
} EDIT. 
tecnología de las empresas. En la consulta desde la encuesta al tema de procesos en el sector empresarial, según el DNP (2010, p. 58-60), se muestran en los resultados obtenidos en el análisis estadístico de la relación entre CIT y desempeño empresarial tres casos: 1) Relación CIT-Desempeño innovador según la primera encuesta; 2) Relación CIT-Desempeño innovador según la segunda encuesta; y 3) Relación CIT-Desempeño del negocio según la primera encuesta.

Por último, los sistemas de información, como la EDIT, para la medición de la innovación que permitan la formulación de políticas, programas y acciones en pos del desarrollo industrial, más que propuesta consolidada en el país para promover la innovación como expresión de las capacidades son un espacio en construcción.

\section{TRANSFORMACIÓN PRODUCTIVA: DESARROLLO PRODUCTIVO Y MERCADOS INTERNACIONALES}

Según Hausmann y Klinger (2006, p. 21) y Berry (1996, p. 105), la transformación productiva sin políticas y con programas débiles y acciones coyunturales sobre los sistemas productivos retarda la incorporación de la innovación. Por un lado, porque los programas de transformación productiva se orientan a la generación de condiciones en sectores productivos específicos, y por el otro, buscan el avance del sector empresarial.

Este proceso se mueve entre dos dimensiones: lo macro (políticas, sistemas y competitividad territorial) y lo micro (dinámica empresarial); estas son importantes en el análisis de la innovación como resultado de las políticas y programas en torno a la transformación productiva. En la primera dimensión se visibiliza la capacidad para asignación de los capitales y las relaciones empresariales como un resultado de las políticas (transformación productiva), de los sistemas productivos y de la capacidad de competitividad territorial. En la segunda dimensión, el mercado aparece como el legitimador de las relaciones productivas desde la empresa, combina la capacidad laboral con las tecnologías y el desarrollo de productos, con los avances en el carácter social y con la capacidad de innovación desde lo público y lo privado.

En este proceso de consolidación de la estructura productiva nacional de Colombia las directrices institucionales son necesarias pero no suficientes. Por un lado, se señala el énfasis en el logro de la transformación productiva desde la innovación para la competitividad y productividad, intencionalidad formulada en la política de desarrollo empresarial de la Ley 1253 de 2008 (CR, 2008); en ella se establece la obligación de presentar un informe sobre el estado de avance de la competitividad nacional. Además, como apoyo a este proceso se ofrecen los incentivos propuestos en la Ley de Pyme, Ley 590 de 2000 y Ley 905 de 2004, la Ley de Fomento al emprendimiento, Ley 1014 de 2006 (CR, 2006) y la Política Nacional 
de Emprendimiento del 2009, las cuales han hecho avances para el desarrollo y fortalecimiento de unidades productivas, la capacitación y el impulso a la asociatividad en búsqueda de la internacionalización, pero falta apoyo directo de las instituciones.

La propuesta institucional es dar cuenta de los cambios regionales y nacional desde el informe que se realiza con base en las directrices formuladas por la entidad rectora de la política, el Ministerio de Comercio, y la política desde los Conpes, los cuales proponen crear una dinámica en el incremento de la productividad y en la generación de más y mejores empleos, para que los sectores aprovechen de forma eficiente las herramientas de internacionalización, con el diseño de estrategias ajustadas a las ventajas comparativas propias y a sus contextos políticos y geográficos (MCIT, 2011).

Articulado a lo anterior, se aprueba el Conpes 3678 de 2010 (DNP, 2010), sobre transformación productiva. La política que sustenta el programa tiene que ver con impulsar la transformación productiva de la economía del país hacia una estructura de oferta diversificada, sostenible, de alto valor agregado y sofisticación que promueva su adecuada inserción en los mercados globalizados y contribuya al mejoramiento de la calidad de vida y el bienestar de los habitantes. Este documento propone dos líneas adicionales de acción: 1) fortalecimiento institucional del Sistema Nacional de Competitividad y 2) acercamiento instrumental a la implementación regional de la política de competitividad y productividad, al enfatizar en las particulares fortalezas y debilidades locales y en la posibilidad de incentivar iniciativas específicas de desarrollo productivo.

Y por el otro, la idea de ampliar las investigaciones sobre factores sociales, culturales y políticos que facilitan y retrasan las actividades innovadoras. En particular, según Sen (2000, p.110), desde las propuestas teóricas de la economía evolucionista y la visión de la empresa basada en recursos y capacidades, un grupo de factores fundamentales que recogen las especificidades explicativas de las dinámicas de una organización están en el concepto de capacidad. Teece, Pisano y Shuen (1997) plantean que las capacidades juegan un papel clave para que la organización adapte, integre y reconfigure habilidades organizacionales, recursos y competencias funcionales en un ambiente cambiante, para el logro de sus objetivos misionales.

La relación entre capacidades y desempeño organizacional queda así planteada como una explicación estructural al comportamiento de las empresas. En particular, según Yoguel, Borello y Erbes (2009), la literatura especializada llama la atención sobre la importancia de aquellas capacidades organizacionales relacionadas con las dinámicas de innovación tecnológica que, por extensión, se denomina capacidades de innovación tecnológica (CIT). 
Es la innovación parte de la construcción social del tejido productivo y, a la vez, el resultado de la articulación entre capacidades humanas, desarrollo de productos y mercados. Por eso, para comprender la propuesta de transformación productiva para el país, es necesario considerar los mecanismos alternativos de diferenciación de iniciativas de desarrollo productivo nacional, departamental y local. Además, se ha detectado que los instrumentos de desarrollo empresarial carecen de la flexibilidad necesaria para adecuarse a las particulares necesidades del desarrollo productivo local para la internacionalización. Además, según DNP $(2008$, p. 4) "... se propone a las entidades del Sistema Nacional de Competitividad una serie de iniciativas de política que se considera mejorarian sustancialmente la competitividad nacional y el ambiente de los negocios...".

En el recorrido por los Conpes elaborados desde el 2006 al 2010, se reconoce la preocupación por pensar políticas estructurales para el desarrollo productivo, y las directrices en el plan de acción de la política de transformación productiva se expresan en el diseño de un programa hasta el 2014 con tres ejes fundamentales, que según MCIT (2011, p. 1) serían: a) promover el avance de sectores nuevos y emergentes de clase mundial; b) estímulo a la producción de los sectores actuales a través de la agregación de valor y la innovación; y c) la promoción de la adopción de las mejores prácticas internacionales en gestión asociativa y gremial.

El contexto del programa de transformación productiva es débil, aunque la formulación busca fortalecimiento estructural. La propuesta se orienta con énfasis en los sectores seleccionados desde la política de competitividad, sectores establecidos y sectores emergentes con potencial de alto valor agregado. Además, se identificaron ocho sectores: Cosméticos y Aseo; Aparatos Domésticos; Autopartes; Farmacéuticos; Tercerización de Servicios [BPOEO $]^{5}$; Desarrollo de Software y Servicios de Tecnologías de la Información; y Salud y Turismo de Salud, los cuales aún son incipientes pero tienen alto potencial de generación de valor agregado, capacidades y una dinámica de crecimiento importante en el contexto mundial. En esta selección se identifica una visión estructural de largo plazo, el problema es que se incluye solo textil-confección-diseño-moda del sector manufacturero; y el resto corresponden al sector servicios.

En estas estrategias que buscan mayor inserción en los mercados internos y externos, se han definido cinco áreas estratégicas de trabajo, como son: modificaciones institucionales, implementación de reformas, consolidación y difusión de programas existentes, simplificación y adecuación de los trámites de comercio exterior a estándares internacionales y de fortalecimiento para la participación de

Esta sigla, por su significado en inglés (Business Process Outsourcing and Offshoring) se refiere a la tercerización de servicios a distancia, definida así según documento elaborado por USAID y Mincomercio (2008). 
Colombia en foros internacionales de Competitividad (DNP, 2008, p. 26; Hausmann y Klinger, 2006; Krugman, 1991, p. 485).

De acuerdo con el documento Conpes 3668 de 2010 (DNP, 2010), llevar a cabo un seguimiento al programa es esencial pues se propone un ejercicio obligatorio de rendición de cuentas a la política pública de competitividad y productividad; es, a la vez, una oportunidad para realizar necesarios ajustes en la dirección de fortalecer la ejecución de la política, sobre todo cuando esta ha sido concebida en un horizonte de largo plazo y, por tanto, requiere continuidad. Además, el plan de acción para la Productividad y el Empleo, DNP (2008, p. 23-26), tiene como objetivo permitir a las empresas colombianas mejorar su capacidad de inserción tanto en el mercado nacional como internacional, al generar un impacto positivo en el empleo y en la calidad de vida de los colombianos. En la búsqueda de ese gran objetivo se trabajó en cuatro ejes estratégicos: 1) emprendimiento, 2) productividad y crecimiento, 3) infraestructura de la calidad y 4) Capacitación y divulgación.

Los estímulos a la producción se establecen con miras a mercados de productos de talla mundial. Esto requiere recurso humano calificado y capacitado para el desarrollo de innovaciones en las empresas, en especial en las pymes. En efecto, los mayores niveles de comunicación que se establecen, los menores niveles de formalización (flexibilidad) que dotan de una mayor autonomía a los trabajadores, y el mayor compromiso que se establece entre trabajador y empresa facilitan la participación de estos en el desarrollo productivo con innovaciones (Hendricks y Singhal, 1997).

Con la Ley de Ciencia y Tecnología, Conpes 3582 del 2009 (DNP, 2009), se sugieren las directrices para el desarrollo empresarial, y en los Conpes se consolidan estrategias de acción.

Por último, se busca mayor internacionalización. El programa de transformación productiva con la inversión en tecnología, tiene limitación por el recurso existente, esto hace que muchas veces en las empresas sea imposible la creación de departamentos de I+D+i (investigación, desarrollo e innovación), por lo que la cooperación entre las pymes vuelve a convertirse en un arma estratégica al generar redes colectivas de conocimiento que sirven de base al desarrollo de las mejoras en productos, servicios o procesos (Verhees y Meulenberg, 2004). Además, sobresale la asociación entre el desempeño innovador y la capacidad de Producción, temas que la EDIT considera fundamentales en la medición I+D.

Los alcances de la transformación productiva miden los resultados de la encuesta EDIT, y es desde ellos desde donde se definen las políticas y el modelo de desarrollo sustentado en el fortalecimiento empresarial porque plantean que la actividad innovadora promovida por las grandes empresas e inhibida para las pe- 
queñas es el soporte y jalonamiento de su desarrollo; por eso se encuentra que los estudios para cuantificar el desarrollo y los procesos innovadores solo se realizan en las grandes (Acs, Audretsch y Feldman, 1994); mientras que Scherer(1980) afirma que es equivocada la búsqueda de un tamaño de empresa ideal para la innovación.

Además, la innovación consiste en un proceso de aprendizaje que se produce como consecuencia del ejercicio de las capacidades productivas y empresariales, en general, y de aquellas que proporcionan el uso de los bienes y servicios producidos. Schumpeter (1978) planteaba que el desarrollo económico está movido por la innovación, a través de un proceso dinámico en el cual nuevas tecnologías sustituyen a las antiguas (OECD 2006).

En este proceso, el avance de las firmas se centra en factores que pueden ser usados en provecho de lograr las metas y objetivos propuestos por las empresas. Entre estas se destaca, según Acs, Audretsch y Feldman (1994, p. 2), el desarrollo de nuevos productos, servicios o procesos que den una respuesta satisfactoria a los clientes, pero además que permitan a la empresa adaptarse al cambio. Si bien las grandes empresas cuentan con mayores recursos financieros y humanos, muchas estadísticas muestran que son las pyme las principales dinamizadoras de la innovación, esto es producto de las particularidades que presenta este tipo de empresas, pues al ser pequeñas en tamaño, logran adaptarse en forma rápida a los cambios y tomar decisiones a través de mecanismos flexibles; este conjunto de características hacen que estas empresas sean acreedoras de ventajas competitivas frente a otros que se encuentren en el mercado.

Los países serán cada vez más productivos y competitivos si quieren permanecer tanto en el mercado mundial como en el propio. Para ello, en temas de innovación el énfasis se debe poner en el desarrollo del capital humano mediante el uso de prácticas organizativas que fomenten el flujo de conocimiento entre los empleados, así como instrumentos que permitan consolidar y motivar en la empresa a los actuales empleados.

La socialización de la innovación promueve las capacidades para que los procesos de transformación productiva superen el desarrollo desigual de los territorios y garantiza la sostenibilidad de las formas de organización del trabajo y de la producción acorde con la inclusión en los mercados internacionales. En este sentido, los sistemas productivos se guían por agentes económicos que operan en un marco de creciente competencia, donde se requiere un nivel cada vez mayor de capacidades cognitivas en la sociedad (Yoguel, Borello y Erbes, 2009; Montenegro y Niño, 2001). Estas capacidades no devienen sólo de factores estáticos dados, sino que se ha acrecentado la importancia del clima institucional en que operan las empresas en el contexto social. Así, la orientación de las políticas hacia las empresas pyme se 
constituye en una fuente creciente de generación de unidades productivas con empleo de calidad y niveles de ingreso que garanticen la inserción en los mercados nacionales e internacionales.

La innovación es un referente del derecho al desarrollo. Braudel (1984) plantea que por encima de los mercados, se sitúa, en manos de actores brillantes, una poderosa superestructura de intercambios. El mercado es uno de los mecanismos mayores de la internacionalización de la economía; su desarrollo es el eje del capitalismo. Las estrategias que se promueven hoy tienen como objetivo el logro de la transformación productiva, en especial de las pymes para sostenibilidad con la productividad y competitividad.

En esa medida, Colombia a través de la propuesta de transformación productiva, busca garantizar su inserción en los mercados internacionales e internacionalizarse. Según DNP (2008, p. 19): "la estrategia adoptada por el gobierno incluye cinco ejes estratégicos determinantes para la competitividad: 1) Atracción de inversión, 2) Regulación propicia para la inversión, 3) Diálogo público-privado, 4) Acuerdos comerciales y de inversión, 5) Simplificación de la regulación de comercio exterior".

El programa de transformación productiva se sustenta en un modelo productivo que incluya delineamiento de diversificación, productividad y mercado exportador orientado desde planificación inteligente, planes excelentes y esfuerzos enfocados. No puede darse transformación productiva sin antes definir el modelo productivo sectorial con directrices a la innovación.

La internacionalización de la economía colombiana desde los procesos productivos, en el siglo XXI, incorpora políticas de competitividad diseñadas para la transformación productiva con innovación, fortalecimiento de capacidades y el desempeño empresarial. Este reto exige desde la perspectiva de los recursos y capacidades, que detrás de la innovación como proceso empresarial estén las capacidades organizacionales. De acuerdo con Acs, Audretsch y Feldman (1994): "... poco se ha escrito en la literatura económica sobre la identificación de aquellas condiciones y contextos de mercado que son propicios para la actividad innovadora".

Existe un gran interés por estudiar la relación entre capacidades y desempeño empresarial, en la medida en que las políticas y estrategias en la nación, en el sector y en la empresa, contribuyen a generar procesos de acumulación, en especial desde las habilidades (Robledo, Gómez y Restrepo, 2008, p. 1).

A manera de ejemplo, en el caso de Colombia se presenta una alta concentración de empresas de todos los tamaños, que evidencia la existencia de importantes brechas de desarrollo entre las regiones. Según DNP (2007, p. 2), cinco departamentos concentran el $79 \%$ de las grandes empresas y el $62 \%$ de las Microempresas y las pyme 
del país; Bogotá 22,8\% 36,1\%. Antioquia 13,5\% 17,9\%. Valle 9,6\% 9,2\%. Cundinamarca 6,3\% 7,3\%. Santander 5,9\% 3,5\%. Atlántico 4,2\% 5,2\%. Resto del país 37,8\% 20,8\%.

La estrategia central para la transformación productiva está en la promoción de las micro, pequeñas y medianas empresas, y la política de emprendimiento. Además, la formulación de la Ley de Pyme (CR, 2000) busca un trabajo concertado con las diferentes instituciones del gobierno comprometidas con el desarrollo de las microempresas y las pymes, las organizaciones representativas del sector privado, los consejos superiores de la microempresa y de la pymes, el sector académico y algunos organismos de cooperación internacional (DNP, 2007, p.18).

De acuerdo con lo anterior y con autores como Acs, Audretsch y Feldman (1994; 1996), se identifican al menos tres líneas de profundización a seguir para la investigación empresarial en el corto plazo: 1) Consolidar los resultados de los estudios desde la doble perspectiva analítica y procedimental, con revisión crítica las definiciones de los conceptos relativos a las capacidades y los desempeños, la selección de las variables observables que los componen, las métricas establecidas para las mismas y las técnicas estadísticas aplicadas. 2) Replicar los análisis para empresas de una misma división territorial y de un mismo grupo industrial y comparar los resultados. 3) Escalar los resultados del grupo industrial. Ahora bien, en el caso de esta última línea de profundización el escalar los resultados se ven reflejados en los tres dígitos del código CIIU, además de la integración de datos complementarios obtenidos por el DANE (2005).

A manera de síntesis, la disminución de las disparidades y desigualdades requería de la implementación y seguimiento de los lineamientos establecidos en el Plan Nacional de Desarrollo 2006-2010, DNP (2007), para el fortalecimiento empresarial. Este plan se estructuró alrededor de nueve líneas estratégicas interdependientes y complementarias: 1) la facilitación del acceso a servicios financieros; 2) el fomento a la formalización de la actividad empresarial; 3) el fomento al desarrollo del mercado de servicios no financieros de desarrollo empresarial (SDE); 4) el fortalecimiento de la capacidad de innovación y la transferencia de tecnología; 5) la promoción del uso de TIC; 6) el acceso a la formación para el trabajo; 7) la facilitación del acceso a mercados; 8) el fomento del emprendimiento; y 9) la promoción de la articulación productiva y la asociatividad empresarial.

La innovación como estrategia desde el programa de transformación productiva, con socialización, constituye una propuesta sugerente cuando se busca el derecho al desarrollo. Las políticas buscan mejoras sostenibles de la productividad y competitividad de las pymes, como una fuente creciente de generación de ingresos y empleo de calidad, y que logren insertarse y posicionarse en los mercados nacionales e internacionales. En los planes y programas se encuentra que los efectos en la 
estructura productiva han sido desiguales en cuanto a la implementación y resultados de la política: en la composición (pequeñas, medianas y grandes empresas), combinación de factores (capital y trabajo) y en capacidad para la innovación.

\section{CONCLUSIONES}

Las transformaciones en el sistema productivo, en las últimas dos décadas, se han visto afectadas por los cambios en el escenario internacional, uno de ellos es el incentivo a la inversión extranjera. Además, de acuerdo con Yoguel, Borello y Erbes (2009) los nuevos paradigmas tecno-organizacionales, la globalización de los mercados, la volatilidad de la demanda, los cambios producidos en las tecnologías de la información y comunicación y los nuevos escenarios han aumentado la competencia y, por ende, la exigencia hacia la competitividad.

La ausencia de un modelo productivo sectorial en el cual la transformación productiva con competitividad e innovación sea explícita es parte del problema hoy. La centralidad de la transformación productiva se orienta a procesos innovadores pero tiene dificultades en las articulaciones empresariales. Aquellas empresas que solo realicen nuevas inversiones cuando comprueben que los competidores obtienen buenos resultados -o bien no posean información fiable de la posición tecnológica de las empresas rivales- estarán abocadas al fracaso.

El fortalecimiento de las estructuras productivas, desde la transformación productiva con innovación, pasa por decisiones de políticas que garanticen la inserción de las instituciones y el país en la internacionalización; la apertura a los mercados globales y locales cuando se dirigen ventajas competitivas en sistemas territoriales desde la reformulación y adaptación de los conocimientos para diferenciación.

Asimismo, el desarrollo de procesos de aprendizaje y el incremento de la capacidad innovadora, son conceptos centrales en el tránsito de las ventajas comparadas estáticas a las ventajas competitivas dinámicas, porque les incluye un rol fundamental para acceder al conocimiento tecnológico y organizacional.

A continuación se expone un conjunto de retos que le permiten a las empresas y al territorio desarrollar nuevas ideas o que haya acceso a dicho conocimiento:

- Retos de los aprendizajes institucionales y empresariales: las capacidades se desarrollan a través de la creación de un modelo educativo con innovación para aumentar las capacidades tecno-productivas. Así, en el nuevo escenario se tornan más importantes para la creación de ventajas competitivas las respuestas de las firmas que apuntan a idear, efectuar desarrollos y mejoras de productos y procesos, efectuar cambios organizacionales y desarrollar nuevas formas de vinculación 
con el mercado. Es decir, en la búsqueda de diferenciación, implícita en el proceso de competencia, los agentes se orientan a aumentar su capacidad innovadora.

- Retos en la transformación productiva: las políticas orientadas al desarrollo de sectores de clase mundial con tecnologías y sistemas productivos innovadores para avance de las empresas es el eje que guía esta propuesta.

- Retos económicos: en este punto se considera el conjunto de conocimientos, rutinas y habilidades tecnológicas y organizativas en la producción y el mercado. Dentro de los recursos tecnológicos se incluyen el stock de tecnologías, la experiencia en su aplicación (know-how) y los medios humanos, científicos y técnicos para su desarrollo. Para afrontar los abundantes desafíos del entorno económico y poder adaptarse a las exigencias de los clientes, las unidades productivas, en este caso las pyme, examinarán de forma cuidadosa las mejoras que pueden introducir tanto en sus productos o servicios como en los procesos.

El reto económico tiene que ver con crear y fortalecer sistemas locales de innovación que incluyen tanto los sistemas productivos como las instituciones educativas y de formación, los sindicatos y asociaciones de profesionales y las entidades de investigación y desarrollo tecnológico involucradas en la transformación productiva.

Además, la apropiación de las capacidades tecno-productivas y el aprovechamiento de las ventajas que ofrece el territorio y la potencialidad que este puede generar a las firmas son vitales para el crecimiento industrial y la consolidación de las pyme. La flexibilidad y los procesos innovadores generan dinámicas que propician optimización y eficiencia en la combinación de los factores productivos.

- Retos en la socialización de la innovación. Para ello, incorporar o desarrollar activos tecnológicos que les permitan posicionarse por delante de su competencia. Las actividades innovadoras constituyen condición necesaria pero insuficiente para garantizar un buen comportamiento económico, en especial de las unidades productivas.

La sociedad de información promueve la facilidad de acceso y promoción de la actividad innovadora. El diseño industrial y su desarrollo está marcado por los componentes de I+D+I y el conocimiento, que conforman actividades productivas de alto dinamismo y marcan la necesidad de conocer los movimientos tecnológicos que se dan en el mercado, tanto en el ámbito internacional como en su propio entorno.

- Retos culturales: la innovación como base del cambio tecnológico es un asunto cultural y, a la vez, requiere un plan de convertibilidad de las empresas acorde con la producción de bienes y servicios en los mercados locales internacionales. La vigilancia tecnológica se ocupa de las tecnologías disponibles o que acaban de aparecer, capaces de intervenir en nuevos productos o procesos. 
La cultura de innovación y cultura empresarial ayudan a un modelo de desarrollo más equitativo, a través del fortalecimiento de los valores de solidaridad, justicia y compromiso con espíritu asociativo.

- Retos ambientales: la dinámica de transformación productiva que garantice el desarrollo sostenible con competitividad e innovación involucra la necesidad de estar al tanto de los avances y tendencias para tener un riguroso seguimiento del mercado es cada vez más latente, a través de identificar segmentos, medir su dinamismo, identificar oportunidades y también amenazas, desde el punto de vista ambiental.

En términos generales puede hablarse del plano global y del plano local de la interacción económica y el fortalecimiento productivo. Se promueve desde la política de transformación productiva la potenciación de la capacidad de las firmas para articular conocimientos generados en ambos entornos, con el consecuente comercio con procesos de apertura y cierre a las interacciones con el espacio global de firmas y sistemas locales.

En definitiva, la innovación es un proceso de las empresas que se incorpora de manera particular y social en el territorio. Se trata de procesar conocimiento desde las organizaciones para anticiparse a los cambios desde las innovaciones y los procesos productivos. Cabe seguir preguntándose: 1) ¿Hasta dónde el modelo tecno-productivo ha logrado la innovación para la internacionalización?; 2) ¿Cuáles son los mecanismos de movilidad de recursos entre pyme que garanticen la inserción de la innovación en la transformación productiva?

\section{BIBLIOGRAFÍA}

Acs, Zoltan; Audretsch, David y Feldman, Maryann (1994). R and D spillovers and recipient firm size. En: Review of Economics and Statistics, Vol. 100 No. 2, p. 336-367.

Audrestch, David y Feldman, Maryann. (1996). R and D spillovers and the geography of innovation and production. En: The American Economic Review, Vol. 86 No. 4, p. 630-639.

Berry, Albert (1996). Creación de un medio normativo propicio para la promoción de la pequeña empresa, perspectivas tradicionales e innovadoras. En: Investigación Económica, No. 217, p. 11-48.

Braudel, Fernand (1984). Civilización material, economía y capitalismo. Madrid, Alianza Editorial, Vol. 3, 560p.

Cardona, Marleny y Osorio, Ana Rocío (2003). La gestión empresarial del desarrollo de las transferencias tecnológicas, Las Redes y las Competencias Laborales. Informe de Investigación, EAFIT, Medellín, 230p.

Castell, Manuel y Hall, Peter (2001). Tecnópolis en el Mundo. La información de los complejos industriales del siglo XXI. Madrid, Alianza editorial, 363p. 
Castells, Manuel (2002). La era de la información, economía, sociedad y cultura, volumen 1: la sociedad red. México D.F., Siglo XXI editores, 592p.

Christensen, Jens Frøsle (1995). Asset profiles for technological innovation. En: Research Policy, Vol. 24, No. 5, p. 727-745.

Cohen, Wesley y Kepler, Steven (1996). A Reprise of Size and R and D. En: Economic Journal, No. 106, p. 925-951.

CR - Congreso de la República - (2000). Ley 590. Por la cual se dictan disposiciones para promover el desarrollo de las micro, pequeñas y medianas empresa. Bogotá, Gaceta oficial, 10 de julio de 2000, p. 1-15.

CR - Congreso de la República - (2004). Ley 905. Por medio de la cual se modifica la Ley 590 de 2000 sobre promoción del desarrollo de las micro, pequeña y mediana empresa colombiana y se dictan otras disposiciones. Bogotá, Gaceta Oficial, agosto 2 de 2004, p. 1-10.

CR - Congreso de la República- (2006). Ley 1014. De fomento a la cultura del emprendimiento. Bogotá, Gaceta oficial, 26 de enero de 2006, p. 1-10.

CR -Congreso de la República- (2008). Ley 1253. Por la cual se regula la productividad y competitividad y se dictan otras disposiciones. Bogotá, Gaceta oficial, 27 de noviembre de 2008, p. 1-2.

CPC -Consejo Privado de Competitividad- (2011). Informe Nacional de Competitividad. Capítulo 9 Sistema Tributario. Bogotá, CPC, 304p.

DANE (2005). Encuesta Anual Manufacturera. [En línea] DANE, Bogotá, http://www.dane.gov.co/ index.php?option $=$ com _ contentEview $=$ articleEid $=96 \mathcal{E}$ Itemid $=59$. $[10 / 10 / 2011]$.

DIAN (2008). El gasto tributario en Colombia. Principales beneficios en el impuesto sobre la renta e IVA. Años gravables 2006 y 2007. [En líneal DIAN, Bogotá, UAE-DIAN, septiembre de 2008, http://www.estimacionestributarias.com/archivos/colombia.pdf. [30/10/2011].

DNP (2007). Política Nacional para la transformación productiva y la promoción de las micro, pequeñas y medianas empresas. Documento Conpes 3484. Agosto 13 de 2007.

DNP (2004). Propuesta para modificar el actual régimen de inversión extranjera contenido en el decreto 2080 de 2000. Documento Conpes 3322. Diciembre 14 de 2004.

DNP (2007a). Plan Nacional de Desarrollo 2006 2010: Estado Comunitario Desarrollo para Todos. Tomo I. Departamento Nacional de Planeación. Bogotá, 440p.

DNP (2008). Política Nacional de Competitividad y productividad. Documento Conpes 3527. Agosto 23 de 2008.

DNP (2009). Política Nacional de Ciencia Tecnología e Innovación. Documento Conpes 3582. Abril 27 de 2009.

DNP (2010). Política Nacional para la transformación productiva: Un modelo de desarrollo sectorial para Colombia. Documento Conpes 3678. Julio 21 de 2010.

DiBella, Anthony; Nevis, Edwin y Gould, Janet (1996). Understanding Organizational Leaning Capability. En: Journal of Management Studies, Vol. 33, No. 3, p. 361-379. 
Marleny Cardona A. - Santiago Escobar A.

Dosi, Giovanni (1982). Technological Paradigms and Technological Trajectories, En: Research Policy, No. 11, p. 147-162.

Duran, Xavier; Ibáñez, Rodrigo; Salazar, Mónica y Vargas, Marisela (1998). La innovación tecnológica en Colombia: características por tamaño y tipo de empresa. Bogotá, Departamento Nacional de Planeación, 237p.

Galindo, Arturo y Meléndez, Marcela (2011). La triste historia de los estímulos tributarios en Colombia. [En línea] Blog Foco Económico, Buenos Aires, Foco Económico, 31 de mayo de 2011, http://focoeconomico.org/2011/05/31/la-triste-historia-de-los-estimulos-tributariosen-colombia/. [3/12/2011].

Guan, Jianch y Ma, N. (2003). Innovative capability and export performance of Chinese firms, En: Technovation, Vol. 23, No. 9, p. 737-747.

Hausmann, Ricardo y Klinger, Bailey (2006). Structural Transformation and Patterns of Comparative Advantage in the Product Space. En: CID Working Paper, No. 128, agosto, p. 54-76.

Hendricks, K. B. y Singhal, V. R. (1997). Does Implementing an Effective TQM Program Actually Improve Operating Performance? Empirical Evidence from Firms that have Won Quality Awards. En: Management Science, Vol. 43, No. 9, septiembre, p. 1258-1274.

Krugman, Paul (1991). Increasing Returns and Economic Geography. En: The Journal of Political Economy, Vol. 99, No. 3, Jun, p. 483-499.

Lam, Alice (2002). Los Modelos societales alternativos de aprendizaje e innovación en la economía del conocimiento. [En línea] Organización de Estados Iberoamericanos, Madrid, OEI, http:// www.campus-oei.org/salactsi/lam.pdf. [10/08/2011].

McEvoy, G. M. (1984). Small business personnel practices. En: Journal of Small Business Management, octubre, Vol. 22, No. 4, p. 1-8.

MCIT -Ministerio de Comercio, Industria y Turismo - (2011). ABC Programa de Transformación Productiva. [En línea], Mincomercio, Bogotá, 4 de junio de 2011, https://www.mincomercio. gov.co/publicaciones.php?id=18033. [1/07/2011].

Montenegro, Santiago y Niño, Luis Carlos (2001). La Tecnología de la Información y de las Comunicaciones en Colombia Proyecto Andino de Competitividad. Documentos de trabajo. Bogotá, Centro de Estudios de Desarrollo Económico Facultad de Economía Universidad de los Andes, 86p.

Neffa, Julio (2000). Las innovaciones científicas y tecnológicas. Una introducción a su economía política. Buenos Aires, Trabajo y Sociedad -CEILPIETTE/CONICET- Lumen-Humanitas, 402p.

OCDE (2006). Manual de Oslo: Guía para la recogida e interpretación de datos sobre innovación. París, Grupo Tragsa (3 ed.), 188p.

Robledo, Jorge; Gómez, Fredy y Restrepo, Juan Felipe (2008). Relación entre capacidades de innovación tecnológica y desempeño empresarial en Colombia. En: Memorias I Congreso Internacional De Gestión Tecnológica E Innovación. Bogotá, Cargraphics, Vol. 1, Fasc. 1, 112p.

Sen, Amartya (2000). Desarrollo y Libertad. Barcelona, Editorial Planeta, 440p. 
Scherer, Frederic M (1980). Industrial market structure and economic performance. Chicago, Houghton Mifflin (2 ed.), 632p.

Schumpeter, Joseph (1978). Teoría del desenvolvimiento económico. México D.F., Fondo de Cultura Económica, 255p.

Teece, David J.; Pisano, Gary y Shuen, Amy (1997). Dynamic capabilities and strategic management. En: Strategic Management Journal, No. 18, agosto, p. 509-533.

USAID y Mincomercio (2008). Desarrollando el Sector de BPOEO como uno de Clase Mundial. [En línea] USAID Y Mincomercio, Bogotá, Mckensey and Company, octubre de 2008, https:// www.mincomercio.gov.co/ptp/publicaciones.php?id=18292. [30/11/2011].

Veltz, Pierre (1999). Mundialización, ciudades y territorios. Barcelona, Editorial Ariel, 254p.

Verhees, Frans y Meulenberg Michiel T. (2004). Marker orientatión, innovativeness, product innovation and perfomace in small firms. En: journal of small business management, Vol. 42, No. 2, p. 134-154.

Yoguel, Gabriel, Borello; José A. y Erbes, Analía (2009). Argentina: cómo estudiar y actuar sobre sistemas locales de innovación. En: CEPAL, No. 99, p. 65-82.

Yam, Richard C.M.; Guan, Jian Cheng; Pun, Kit Fai y Tang, Esther P. Y. (2004). An audit of technological innovation capabilities in Chinese firms: some empirical findings in Beijing, China. En: Research Policy, Vol. 33, p. 1123-1140. 